\title{
Computer Simulation of Soft Matter at the Growth Front of a Hard-Matter Phase: Incorporation of Polymers, Formation of Transient Pits and Growth Arrest
}

\author{
Richard P. Sear ${ }^{a}$
}

May 4, 2012

\begin{abstract}
Biominerals are typically composites of hard matter such as calcite, and soft matter such as proteins. There is currently considerable interest in how the soft matter component is incorporated into the hard matter component. This would typically be a protein that does not fold up into a single rigid domain but is closer to a simple polymer, being incorporated into a growing inorganic crystal in aqueous solution. Here I use computer simulation to study a very simple (2D lattice gas) model of a growing phase and a polymer. This allows me to study the microscopic dynamics of incorporation or rejection of a single polymer by the growing phase. It also allows me to look at how high concentrations of absorbing polymer can both arrest crystal growth, and change the shape of crystals. I find that the incorporation of a single polymer into the growing phase is due to slow dynamics of the polymer at the growth front. These slow dynamics are then unable to keep up with the advancing growth front. This is an intrinsically far-fromequilibrium process and so occurs even when incorporation is thermodynamically highly unfavourable. During the incorporation process, large polymers create large and deep, but transient, pits in the growth front.
\end{abstract}

\section{Introduction}

Our bones and teeth are composites of soft matter and hard matter. The soft matter is proteins such as collagen and the hard matter is hydroxyapatite crystals. Bones and teeth are just two examples of a huge range of biominerals that are composites of an inorganic crystal such as hydroxyapatite or calcite, and proteins ${ }^{1}$. Soft and hard matter are very different. Soft matter is as the name suggests rather soft, the effective modulus for compressing a polymer in solution may be $\mathrm{kPa}$. By contrast crystals like hydroxyapatite and calcite have GPa moduli, see Table 1. Soft matter also has characteristic length-scales that can be tens of nanometres or even larger. Inorganic crystals have lattice constants of a few Ångstroms. Many interactions in soft matter are relatively weak, of order the thermal energy $k T$ or less, while the ions in highly insoluble inorganic crystals are held in their lattices by strong, many $k T$, interactions.

These very large differences in properties between soft and hard matter are expected to be reflected in near total immiscibility of soft and hard matter at equilibrium. However, biominerals are formed far from

\footnotetext{
$\dagger$ Electronic Supplementary Information (ESI) available: 6 Movies of the dynamics are available. See DOI: 10.1039/c000000x/ ${ }^{a}$ Department of Physics, University of Surrey, Guildford, Surrey GU2 7XH, United Kingdom. Tel: +44(0)1483 686793; E-mail: r.sear@surrey.ac.uk
} 


\begin{tabular}{|c|c|c|}
\hline & $\begin{array}{c}\text { Soft Matter } \\
\text { (Polymer) }\end{array}$ & $\begin{array}{c}\text { Hard Matter } \\
(\text { Crystal })\end{array}$ \\
\hline lengthscale & $R_{G} \approx 10 \mathrm{~nm}$ & $a \approx 0.5 \mathrm{~nm}$ \\
\hline $\begin{array}{c}\text { free energy } \\
\text { cost of }\end{array}$ & $k T / R_{G}^{3} \approx k T /(10 \mathrm{~nm})^{3}$ & $E \approx 100 \mathrm{GPa}$ (elastic compression) \\
volume change & $\approx 1 \mathrm{kPa}$ & $S / a^{3} \approx 10 \mathrm{MPa}$ (crystallisation) \\
& & $\gamma_{X} / R_{G} \approx\left(k T / \AA^{2}\right) / 10 \mathrm{~nm} \approx 10 \mathrm{MPa}$ (cavity) \\
\hline
\end{tabular}

Table 1: Comparison of the typical values for relevant properties of soft and hard matter. Soft matter has larger length scales than hard matter and so lower free-energy densities (and hence moduli). The soft matter object considered is a linear polymer, with radius of gyration $R_{G}$. In order to obtain specific numbers I take $R_{G}=10 \mathrm{~nm}$. The hard matter is a crystal with a unit cell of order $a$ across, and with a Young's modulus $E$; $E$ is approximately $100 \mathrm{GPa}$ for calcite ${ }^{2}$. One of the sides of the unit cell of calcite is $0.5 \mathrm{~nm}$ across. I give three hard-matter free-energy densities. The first is the elastic modulus (top of the three rows). The second is the free-energy driving force per unit volume, for crystallisation (middle row), and the third is the free energy cost per unit volume of creating a cavity of radius $R_{G}$ (bottom row). $S$ is the supersaturation of the solution and $\gamma_{X}$ is the crystal/solution interfacial tension. I take a supersaturation $S \approx k T$, and an interfacial tension $\gamma_{X} \approx k T / \AA^{2} \approx 100 \mathrm{~mJ} / \mathrm{m}^{2}$.

equilibrium and remain there. The soft matter is incorporated into the growing crystal far from equilibrium, and then is trapped. Motivated by this, here I use computer simulation to study the dynamics of a simple model polymer at the growth front of a growing hard-matter phase. I am interested in how the dynamics of the interaction of hard and soft matter, are affected by their very large differences in properties. Note the soft matter dynamics are here are those at the growth front. Although soft matter is expected to be almost totally immiscible with hard matter, soft matter often strongly adsorbs on surfaces. I am also interested in what features of the polymer control whether or not it is incorporated into the growing crystal.

Any incorporation of polymers or other large soft-matter objects such as micelles, into inorganic crystals will only occur due to out-of-equilibrium processes. So to understand incorporation it is essential to understand the dynamics of the process. Here, I use as simple as model as possible: a two-dimensional lattice model. The use of simulation allows me to track every detail of the microscopic kinetics so for the first time we can observe exactly how soft matter is incorporated into a growing hard phase. Observing this detail is not possible in experiment.

In brief, I find that the large size of the polymer results in slow dynamics, and that these slow dynamics are what causes single polymers to be included in the growing phase. The slow dynamics drive the system far from equilibrium and produces a far from equilibrium hard/soft matter composite. By contrast a small weakly interacting oligomer may rapidly reach local equilibrium at the growth front and then it can 'surf' along on the growth front instead of being incorporated into the growing crystal. When the polymer concentration is high many polymers can bind to and hence cover the growth front. Then the slow dynamics of the polymer can also greatly slow down the rate at which a growth front advances.

As far as I am aware, simulations have not previously been performed on the dynamics of the incorporation of polymers into crystals. Although Muthukumar has developed a theory for the effect of polymer adsorption on crystal anisotropy ${ }^{3}$. There is, however, a significant experimental literature on a diverse range of soft matter objects inside crystals. Examples are naturally occurring proteins ${ }^{4-7}$, polysaccharide poly- 
mer gels ${ }^{8-12}$, and copolymer micelles ${ }^{13}$. The crystalline phase in most studies is calcite, but it is a general phenomenon, other crystals can incorporate soft matter objects ${ }^{5,6}$. Crystals can even incorporate colloidal particles ${ }^{14}$.

There are extensive results on the effect of polymers in solution on crystallisation. Both homopolymers ${ }^{10,15,16}$ and copolymers ${ }^{12}$ can bind to growing crystal surfaces, and this binding affects the growth of the crystal. By changing the growth rates of different crystal faces by different amounts, polymers can change the shapes of crystals. Biological polymers, for example peptides ${ }^{17-20}$ and alginates ${ }^{21}$, have been shown in in vitro experiments to also do this. Indeed this is not restricted to ionic crystals, there are peptides that bind to the surface of ice and inhibit the growth of ice crystals ${ }^{17}$.

It is clear that biominerals such as our bones, sea-urchin spines, etc., all have incorporated proteins, and these natural polymers act to control crystallisation ${ }^{1,22}$. Note that in general in experiment (but not here) it can be difficult to distinguish between the effect a polymer may be having on the nucleation stage of crystallisation and its effect on growth. However it is clear that synthetic polymers and proteins can affect crystal growth. They can do so in a way that is specific to one of a substance's polymorphs and to specific faces of a polymorph.

There are also extensive results on the adsorption and desorption of polymers on static, i.e., not growing, solid surfaces, including flat crystalline surfaces like mica ${ }^{23,24}$. Simply because polymers are large objects they usually adsorb very strongly on solid surfaces, i.e., adsorb irreversibly. Indeed, unless the polymer solution is very dilute the polymer molecules adsorb in such numbers that the solid surface is completely covered with polymer. The physics behind this is simple, if the free energy change per monomer is only, for example, $0.5 \mathrm{kT}$, then the free energy of adsorbing a polymer 100 monomers long is $50 \mathrm{kT}$ - more than enough to drive irreversible adsorption.

For surfaces in contact with highly dilute polymer solutions, isolated single polymers can adsorb, typically irreversibly, via a process with rather complex, often far from equilibrium, dynamics $23,25-28$. As adsorption is so strong, desorption of a single polymer is typically studied by pulling the polymer off the surface using an AFM tip. This requires a sustained force of typically tens of $\mathrm{pN}$ or more ${ }^{23}$. The lifetime of a single polymer at a surface will often be too large to measure in experiment. A single polymer on a surface, although it may never desorb, can diffuse on this surface. This diffusion has been studied, and found to be much slower than in the bulk of the solution ${ }^{29-31}$.

At all but high dilutions, a static solid surface will acquire a covering layer of adsorbed polymer. The polymers in this layer may be dynamic ${ }^{26}$, i.e., the polymers will slowly turn over, but coverage will be complete. The expectation would be that such a layer would greatly inhibit crystal growth, so all but very low polymer concentrations should inhibit the growth of any crystal whose surfaces attract the polymers.

The rest of this paper is organised as follows. The next section describes our results for a single polymer interacting with a growth front. The third section contains our results for the effects on growth of a high concentration of polymers at the growth front. The fourth section presents our conclusions, and the final section gives details of the model and of the simulation method. There is supplementary information in the form of 6 Movies that illustrate the dynamics we study here. As the processes we study here are intrinsically dynamic, they are best understood by seeing the dynamics in these Movies. 
A)

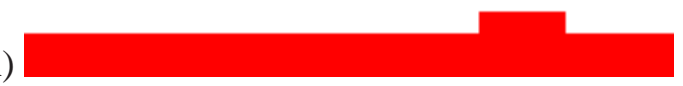

B)

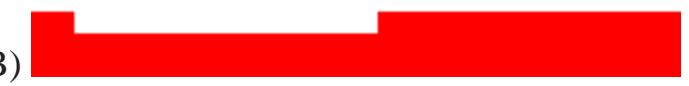

Fig. 1 : Two snapshots of growth in a small system $L=30$ across. These show the growth mechanism of the condensed phase in this model. Empty lattice sites are white, sites filled with particles are red (grey in greyscale image). Snapshot A) shows two complete layers plus the start of a third layer on top of the second complete layer. The third layer is very incomplete, it consists of just 4 particles. In this 2D model the nucleus is a single particle so these 4 particles are over the barrier and will grow. Snapshot B) shows the system 10 cycles later when the third layer has grown so that it is now approximately half complete. 40 cycles later this layer will be complete. The dynamics of this process are shown in Movie 1. The system is at $\varepsilon / k T=9$ and $S / k T=0.2$.

\section{Results for a single polymer}

In this section we will consider a single polymer interacting with a growth front. This will be the case when the polymer solution is dilute. We are interested in what polymer properties determine whether the polymer is incorporated in the growing phase, or is pushed ahead of the growth front. We will vary the length of the polymer, the interaction between the polymer and the growing phase, and the speed of the polymer dynamics.

Our model for the growing crystal, the hard matter part of our system, is a simple two-dimensional lattice gas. It is the lattice gas that can be mapped onto the famous two-dimensional Ising model ${ }^{32}$ solved by Onsager ${ }^{33}$. See section 5 for details of our model. The model has a vapour and a condensed phase. The vapour is our model of the solution and the condensed phase is our model of the crystal. We work at low temperature where the vapour is dilute and in the condensed phase essentially every lattice site is occupied by a particle. This can be seen in simulation snapshots such as those in Fig. 1 where the condensed phase is solid red because lattice sites filled by particles are shown in red, while the vapour is solid white as the density of particles is very low there. I work at constant supersaturation, i.e., the difference in chemical potential between the condensed and the vapour phase, $S$, is constant. The temperature is also constant, and so the ratio of the energy of attraction of two neighbouring lattice-gas particles, $\varepsilon$, and the thermal energy is constant and the same for all simulation runs at $\varepsilon / k T=9$. We mostly work at $S / k T=0.1$ but some runs are at a larger supersaturation of $S / k T=0.2$. Also, simulations are done in a square simulation box of $n_{L}$ by $n_{L}$ sites.

The polymer is a standard linear lattice homopolymer with $L$ monomers. Here we study $L$ between 4 and 256. Each monomer occupies one lattice site, and is shown in green in our simulation snapshots. The polymer is flexible and its dynamics are via individual monomer moves. See section 5 for details of the model polymer. Like the Ising model for the hard matter phase, our model polymer is quite standard ${ }^{34}$. The interaction between a monomer and lattice-gas particle is $\varepsilon_{M}$. We consider values of $\varepsilon_{M}$ in the range $0<\varepsilon_{M}<\varepsilon$. Because $\varepsilon_{M}>0$, the polymer adsorbs on the surface of the growth front - this reduces the interfacial energy of this interface. However, because $\varepsilon_{M}<\varepsilon$ there is an energy cost for incorporation of the polymer into the condensed phase, as then particle-particle bonds are replaced by weaker particle-monomer 


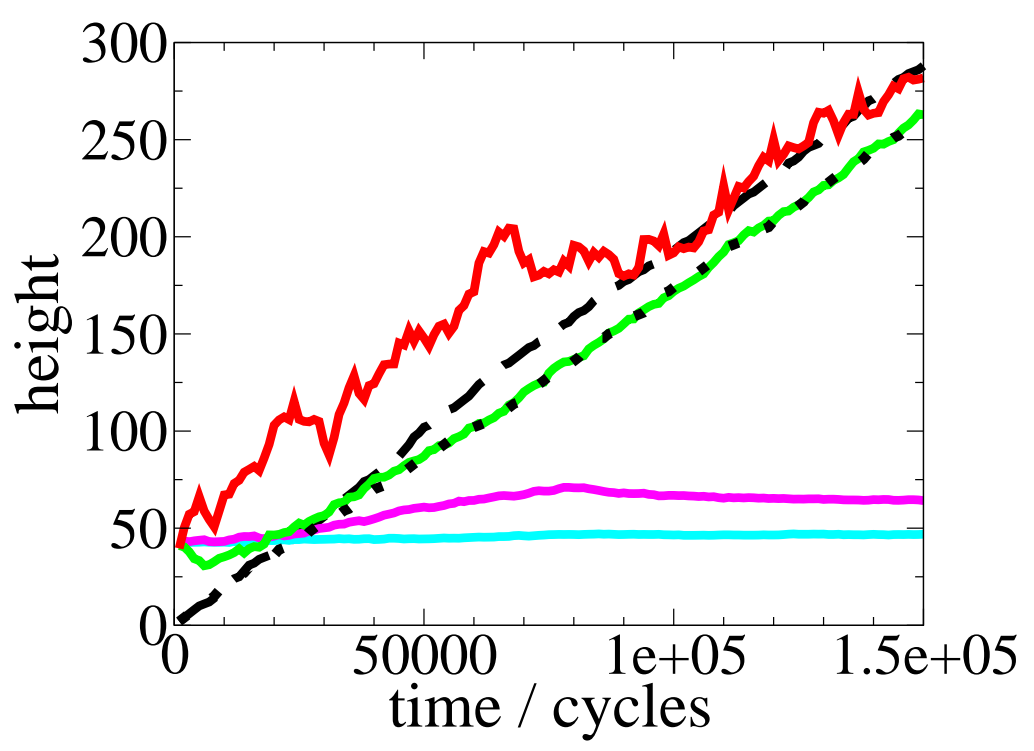

Fig. 2 : Plots of growth fronts (black curves), and the centres of mass of polymers (coloured curves), both as a function of time. The length of the polymer is $L=100$. The growth front is defined as being the height of the top layer that is more than half occupied by lattice-gas particles, in units of the lattice spacing. The black dashed curve is the growth front in the absence of polymer, while the black dotted curve is the growth front in the presence of polymer with $s_{P}=10$. Note that the two growth fronts are almost parallel. The growth fronts for other values of $s_{P}$ are also very similar and so are not shown for clarity. The centres of mass of polymers with $s_{P}=0.1,1,10$ and 100 are shown as cyan, violet, green and red curves, respectively. In greyscale these four curves can be distinguished as at large times, $s_{P}=0.1$ is the bottom curve, then the $s_{P}=1,10,100$ curves are in this order from bottom to top. When the polymer is incorporated into the condensed phase its centre of mass becomes almost static (see the cyan and violet curves), while if it surfs along on the interface its centre of mass tracks the growth front (see the red and green curves). The parameter values are $\varepsilon / k T=9, \varepsilon_{M} / \varepsilon=0.1$ and $S / k T=0.1$, for all polymers, and the box size is $n_{L}=400$.

bonds. So the polymer is essentially a surfactant as it prefers the interface and reduces the interfacial tension.

The model also has an additional parameter, $s_{P}$. This controls the relative speeds of the dynamics of the polymer, and of the dynamics of the condensed-phase. For large values of $s_{P}$ the monomers move rapidly relative to motion of a layer of the condensed phase, while for small $s_{P}$ the monomers move slowly. Changing $s_{P}$ does not change the equilibrium behaviour, so changing $s_{P}$ allows us to distinguish between equilibrium and intrinsically non-equilibrium behaviour. See the Model section, section 5, for details on $s_{P}$, but essentially $s_{P}$ is the ratio between the rate of moves of a monomer and rate at which an attempt is made to add or remove a lattice-gas particle.

\subsection{Growth mechanism of the condensed phase}

We start our calculations by performing simulations of growth without a polymer. Movie 1 shows growth of a few layers in a small $\left(n_{L}=30\right)$ system, and Fig. 1 shows two snapshots from that movie. The position of 


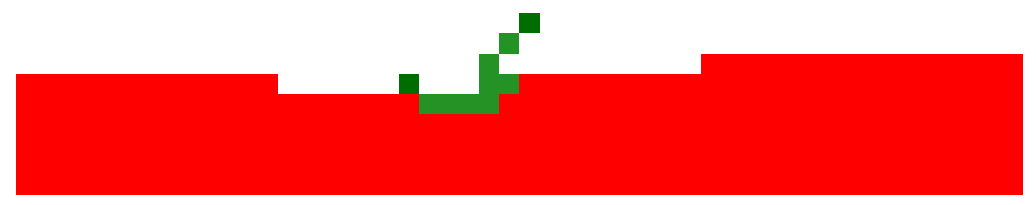

Fig. 3 : A snapshot of a growth front with a polymer of length $L=10$ bound to it. The system is $L=100$ across, but only a part 50 sites wide is shown. Empty lattice sites are white, sites filled with particles are red (dark grey in greyscale), and the monomers of the polymer are green (lighter grey). The monomers at the two ends of the polymer are distinguished by being a darker green than the rest of the monomers. The dynamics of this process are shown in Movie 2. The system is at $\varepsilon / k T=9, S / k T=0.1$, and $\varepsilon_{M} / \varepsilon=0.3$.

the growth front of a larger $\left(n_{L}=400\right)$ system without polymer is shown as the black dashed curve in Fig. 2 . From Fig. 2 we see that the growth rate is approximately $2 \times 10^{-3}$ sites/cycle, for $n_{L}=400$ at $\varepsilon / k T=9$ and $S / k T=0.1$.

From Movie 1 and Fig. 1 we see that the growth front advances single layer by single layer. Each layer first nucleates (in our two-dimensional system the nucleus is a single particle) and then grows laterally. The growth mechanism is nucleation and growth.

Nucleation is an activated process. This is apparent in Movie 1 where many cycles pass before each layer nucleates. Once this single particle is there, growth to the left and to the right does not involve a barrier as the energy cost of adding particles to the left or right of an existing particle is zero. However, growth of the number of particles in an incomplete layer is not monotonic, the number of particles in an incomplete layer undergoes a biased random walk, biased to increase the number of particles by the supersaturation. After a time the layer will either be complete or have dissolved. Then after a waiting time, the next layer will nucleate, and so on. At this low temperature there is a wide range of values of $\varepsilon / k T$ and $h / k T$ over which the growth mechanism is as described here. The mechanism is the two-dimensional version of the conventional crystal growth mechanism where successive layers each form by nucleation followed by growth in the area of the layer ${ }^{35}$.

\subsection{A polymer at the growth front}

Now let us consider a polymer at the growth front. Movie 2 and Fig. 3 show a system with a single short $L=10$ polymer. The monomers of this polymer attract the lattice-gas particles $30 \%$ as strongly as the particles bind to each other: $\varepsilon_{M} / \varepsilon=0.3$. Note that the polymer binds to the surface effectively irreversibly, even though it is short and the attraction between its monomers and the particles is only $30 \%$ as strong 


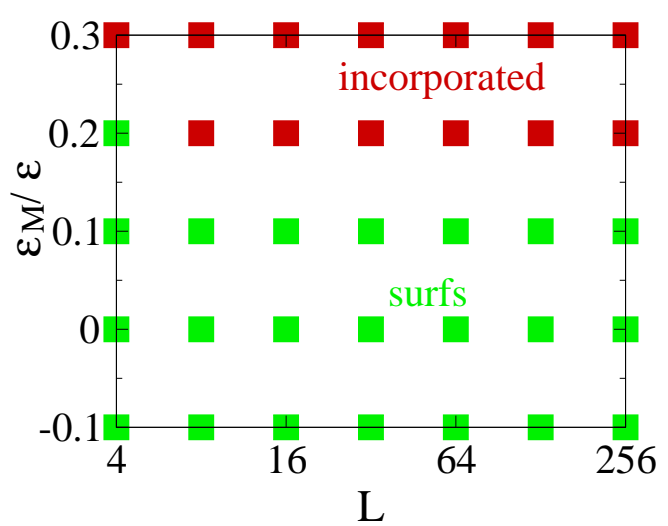

Fig. 4 : Plot of the polymer lengths, $L$, and interaction parameter $\varepsilon_{M} / \varepsilon$ values for which the polymer is incorporated into the growing condensed phase (red squares, or dark grey in greyscale), or surfs along the growth front (green squares, or lighter grey in greyscale). Incorporation is determined for a single polymer at the end of a simulation run of $10^{5}$ cycles. The system is at $\varepsilon / k T=9, S / k T=0.1, s_{P}=10$ and the box size is $n_{L}=400$.

that between the particles of the condensed phase. The total interaction when it lies flat on the surface is $10 \varepsilon_{M}=27 k T$ - enough for essentially irreversible binding. Thus all but very short and weakly interacting polymers will bind to the growth front and essentially never unbind.

Thus for polymers binding to a growth front, the lifetime of binding is much larger than the timescale of adding a layer. This is true even for short polymers of only $L=10$ monomers, and the lifetime of binding will increase exponentially with $L$. Note that it is clear from Movie 2 that individual monomers detach and reattach but these small movements of one or a few monomers do not change the position of the centre of mass of the polymer significantly. Thus motion of the polymer as a whole is very slow in comparison to the rate of advance of the growth front, and so the growth front tends to advance past the polymer.

In Fig. 2 we see that the polymer has essentially no effect on the rate of advance of the growth front. There the dashed black curve is the growth front without polymer, while the dotted black curve is the growth front with a single polymer of length $L=100$. These two curves are almost parallel, which tells us that the growth front is advancing at almost the same rate with and without polymer. This is also true for shorter and for longer polymers, and for all polymer speeds, $s_{P}$ (data not shown).

So, we expect that in experiment the growth rate will not be affected by concentrations of polymer that are low enough that polymer covers only a small fraction of the growth front. This is in systems where growth proceeds via nucleation and growth of new layers. When the polymers cover only a small fraction of the total area, they so do not affect nucleation, and layers can simply grow around them. (Systems that grow via a single spiral defect may be different.)

As a single polymer does not slow growth there are really only two possible things it can do: be incorporated into the growing phase, or 'surf' along on the growth front. See Movie 3 for a polymer being incorporated into a growth front, and Movie 4 for a polymer surfing along on a growth front. For $\varepsilon_{M} / \varepsilon<1$, surfing along on the growth front will be energetically favoured. See section 5.3. 


\subsection{Incorporation of the polymer into the condensed phase}

We have performed a number of simulations in which we varied both $L$ and $\varepsilon_{M} / \varepsilon$. Each simulation was of $10^{5}$ cycles. This is long enough for the growth front to move approximately 175 lattice sites. At the end of each run we checked to see if the polymer had been incorporated into the growing phase, or was surfing along at the growth front.

The results are shown in Fig. 4. For attractions between monomers and particles $\varepsilon_{M} \geq 0.3 \varepsilon$, polymers of all lengths are incorporated. Note that for $\varepsilon_{M}<\varepsilon$, incorporation is energetically unfavourable. Therefore, here incorporation must be due to out of equilibrium kinetics. Also, it is interesting to note that the strength of attraction needed to produce incorporation is relatively insensitive to polymer length. Stronger attractions are needed to force the incorporation of shorter polymers but the increase in $\varepsilon_{M} / \varepsilon$ is small and it is only for very short polymers. This is perhaps unsurprising as for all except very small values of $\varepsilon_{M}$ binding of the polymer to the growth front is irreversible for all except very small values of $L$. For most values of $\varepsilon_{M}$ a bound polymer essentially never unbinds even if it is 10 monomers long.

Finally, it should be noted that Fig. 4 is for runs of length $10^{5}$ cycles, a time long enough for the growth front to advance by approximately 175 sites. If longer runs are done then eventually all polymers are trapped by the growing phase, even ones that surf on the front for shorter runs. Thus the green squares in Fig. 4 do not indicate polymers that are never incorporated, just those that are incorporated very inefficiently. Here, inefficiently means after surfing on on the growth front for at least hundreds of sites of growth, and in most cases much more.

As $\varepsilon_{M} / \varepsilon<1$, it is energetically unfavourable for a polymer to be incorporated into the condensed phase. Thus we suspect that incorporation is due to the system being far from equilibrium. We can test this. Within a simulation model such as ours, it is straightforward to change the speed of the dynamics, while keeping the equilibrium behaviour completely unchanged. If this affects incorporation behaviour then it must be a non-equilibrium effect. We vary the parameter $s_{P}$, that controls the relative rates of attempts to move a monomer, and to occupy/fill a lattice site. We do so while keeping $\varepsilon / k T, \varepsilon_{M} / \varepsilon$ and $S / k T$ all constant, so only the dynamics change. As $s_{P}$ increases, the dynamics of the polymer speed up relative to the dynamics of the growth front.

The results are shown in Fig. 2. The different coloured solid curves in this figure are for $L=100$ polymers that are identical except for the speed of their dynamics. The cyan curve is the centre of mass of the slowest polymer and the red curve is the centre of mass of the fastest polymer. Clearly, the two slower polymers (cyan and violet curves) are incorporated into the growth front, while the faster polymers (green and red curves) move sufficiently fast to surf along on the growth front. Movie 3 shows a polymer being incorporated, while Movie 4 shows a polymer surfing along on the growth front and not being incorporated into the growing phase. The same trend is also seen both for shorter and for longer polymers (data not shown), i.e., decreasing $s_{P}$ slows the polymer and promotes incorporation. In all cases if the dynamics are slow enough the polymer is incorporated.

This supports our hypothesis that polymers are incorporated not because this is the equilibrium state but because the dynamics of motion of the polymer absorbed on the surface is too slow to keep up with the growth front and so the system is forced into the highly non-equilibrium state in which the polymer is incorporated into the condensed phase. In other words, incorporation is a kinetic not an equilibrium effect. If this is general, it implies that for a polymer, micelle, or other soft-matter object, to be incorporated into a growing crystal, all that is needed is to slow the kinetics of the soft matter at the growth front, until they cannot keep up with the growth front. 
A)

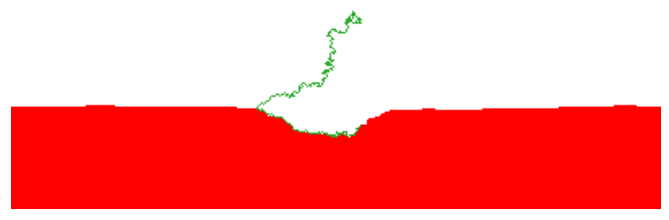

C)

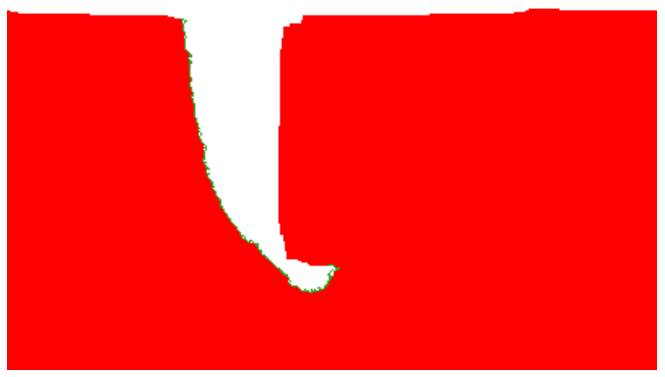

B)

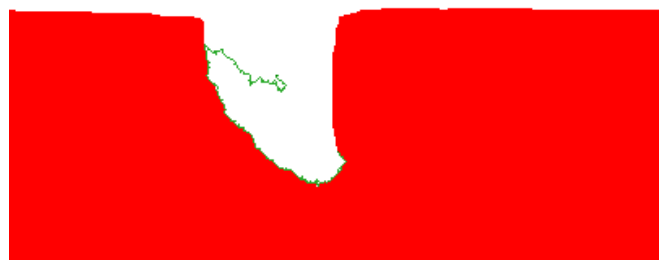

D)

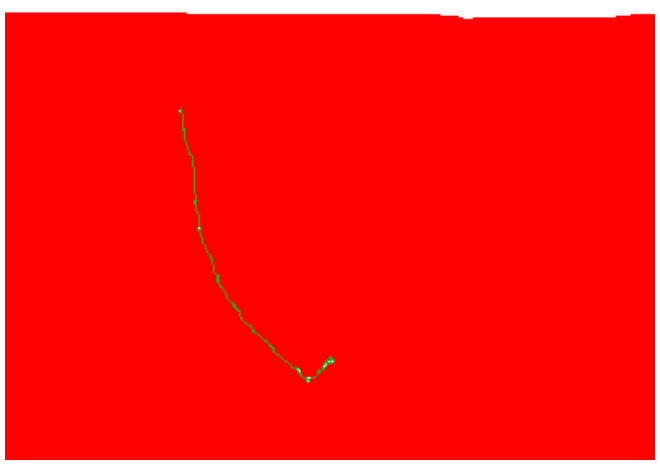

Fig. 5 : Four snapshots of growth and incorporation of an $L=256$ polymer. Empty lattice sites are white, sites filled with particles are red (dark grey in greyscale) and the polymer is green (lighter grey). Snapshot B) is 53,000 cycles after snapshot A), snapshot C) is another 38,000 cycles later, and snapshot D) is 32,000 cycles after snapshot C). Snapshot A) shows the initial binding of the polymer to the surface, and the start of growth around the polymer. Snapshots B) and C) shows the polymer at the bottom of a pit that has formed around it as the growth front grows past the polymer. Snapshot D) shows the polymer in its final position incorporated in the condensed phase after the growth front has passed it by. The dynamics of this process are shown in Movie 3. The parameter values are $\varepsilon / k T=9, S / k T=0.1, \varepsilon_{M} / \varepsilon=0.3$, and $s_{P}=10$.

\subsection{Pit formation}

Movie 3 and Fig. 5 show a growth front advancing on a long, $L=256$, polymer. Note that as the growth front grows around the polymer a transient pit is created in the front. This is created by the front advancing forward but not growing sideways over the polymer until the front is a considerable distance past the 


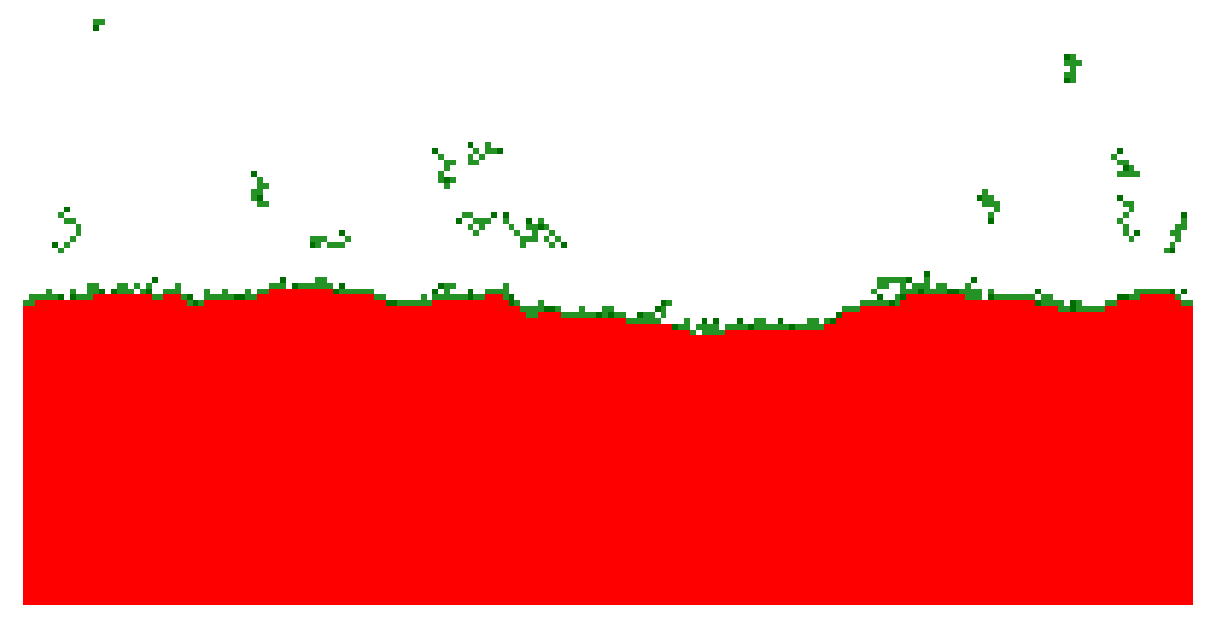

Fig. 6 : Snapshot of a simulation where growth is partially arrested by short, $L=10$, absorbing polymers. There is a high density of polymers: a total of 225 polymers in a square box $n_{L}=400$ sites across. Only a section of $200 \times 100$ sites is shown in the snapshot. The interaction between a monomer and a lattice-gas particle is $\varepsilon_{M} / \varepsilon=0.3$. The supersaturation $S / k T=0.1, s_{P}=10$, and $\varepsilon / k T=9$. See Movie 5 for the growth dynamics; this is the final snapshot from that movie. It is at the end of a simulation run of $10^{6}$ cycles. The lattice-gas particles are red (dark grey in greyscale) and the polymers are green (lighter grey). To make it easier to see where one polymer ends, and another begins, monomers at the two ends of the polymers are darker green than the others.

polymer.

As can be seen in Movie 3 and Fig. 5 the polymer absorbs along a part of the growth front around 100 sites across, blocking growth of this width of the growth front. However, over the rest of the growth front, growth continues. This moves the growth front past the polymer, causing a pit to form. The absorbed polymer is at the bottom of this pit. However, as the depth of the pit increases, the two sides of the pits become growth fronts for lateral growth, i.e., for growth at $90^{\circ}$ to the growth front. The lateral growth of these fronts will eventually cause the pit walls to move together, closing off the pit and irreversibly incorporating the polymer. Because of this lateral growth the pit is only ever transient, ultimately the polymer will always be enclosed on all sides.

\section{Results for a high density of polymers: Growth arrest}

The results of the previous section were all for a single polymer at a growth front that is much larger than the polymer. The single polymer cannot inhibit growth along this front and so is either pushed ahead of the growth front or incorporated into the growing phase. This behaviour will be observed when the polymer is very dilute in solution so that polymer molecules never cover more than a very small fraction of the growth front. In this section we consider what happens when the concentration of polymer is large so that polymers cover a significant fraction of the growth front. We find that the polymers can then greatly inhibit, and even almost completely arrest growth. See Movie 5 for almost completely arrested growth, and the snapshot in Fig. 6 for an almost arrested growth front covered with polymer. 


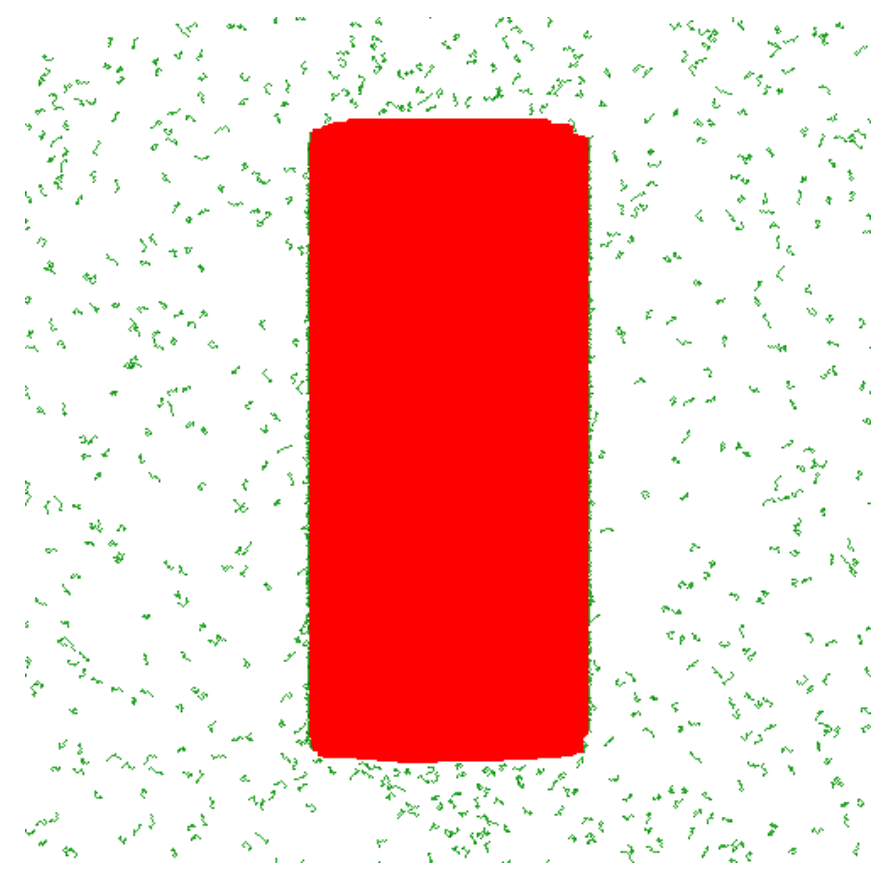

Fig. 7 : Snapshot of a simulation of anisotropic growth of the condensed phase, due to anisotropic binding of the polymer. The simulation is of a box $n_{L}=500$ sites across with 700 short $(L=10)$ polymers that adsorb onto the vertical growth fronts but are repelled by the horizontal growth fronts. The interaction between a monomer and lattice-gas particle is $\varepsilon_{M} / \varepsilon=0.3$ along the horizontal axis but is $\varepsilon_{M} / \varepsilon=-0.3$ along the vertical axis. The supersaturation $S / k T=0.1, s_{P}=10$ and $\varepsilon / k T=9$. The snapshot is after $10^{5}$ cycles. See Movie 6 for the growth dynamics. The lattice-gas particles are red (dark grey in greyscale) and the polymers are green (lighter grey).

In Movie 5 we see growth that is very slow due to adsorbed polymer. Although the growth front is almost completely covered with the polymers, the bond between a monomer and a lattice-gas particle is relatively weak, $\varepsilon_{M}=2.7 k T$. Thus, occasionally monomers lift off from the surface and the condensed phase can grow by a few more lattice-gas particles. This allows the growth front to very slowly advance. Larger values of $\varepsilon_{M}$ greatly slow this.

Studies of a range of values of $L$ and $\varepsilon_{M}$ show that all but short polymers with small values of $\varepsilon_{M}$ adsorb essentially irreversibly on to the growth front. Then, at sufficiently high concentrations these polymers cover the growth front and greatly slow growth. Very short weakly interacting polymers do not bind to the growth front and so do not slow growth.

\subsection{Anisotropic growth due to anisotropic binding}

When polymers bind to growth fronts along one axis but not to fronts along another axis, they can make growth rates anisotropic, and so produce a domain of the condensed phase that is anisotropic. In the absence of polymer or when polymer binds isotropically, symmetry forces the growing condensed-phase domains of our model to be square. Within our model it is straightforward to make binding on horizontal and vertical 
growth fronts different. We simply make the polymer/lattice-gas-particle interaction dependent on direction. For details see section 5.2.

The result is shown in Movie 6 and in Fig. 7. The polymers only bind to the horizontal growth fronts, not to the vertical growth fronts. Thus growth along the horizontal direction is largely arrested while vertical growth is almost unaffected by the polymer and so is much faster. The result is the highly anisotropic domain of the condensed phase shown in Fig. 7. Without polymers the condensed-phase domain would be square.

It is well known that polymers can influence the shape or morphology of crystals, and this is presumed to be as a result of the polymer binding more strongly to some crystal faces than others, therefore affecting the growth rates of different faces by different amounts $5,12,19,36$. In our systems, high concentrations of polymers can almost completely arrest growth on the faces they bind to. The result is highly anisotropic, needle-like domains of the condensed phase.

\section{Conclusion}

Here we have used computer simulation to study polymers at the growth front of a growing phase. Our model is only a very crude model of a crystal such as calcite growing in the presence of polymers, polymer micelles or other soft matter objects. However, we may hope that our generic predictions will hold for most systems of crystals and polymers. For example, we found in our simple model that due to their large size, polymers have slow dynamics, which results in the polymer at the growth interface being very far from equilibrium. In contrast the rapid dynamics of small oligomers allow them to equilibrate at the interface. These slow dynamics of the polymer at the interface then mean that growth occurs around it and the polymer is incorporated into the phase, in a process that is intrinsically very far from equilibrium. This prediction looks rather generic as it just relies on slow dynamics, and real three-dimensional polymers on surfaces have such slow dynamics ${ }^{23,25-31}$. So although there are many differences between our model and experiments, because the effect is so generic, we expect that in both cases incorporation can be driven by the slow dynamics of the soft matter at the growth front failing to keep up with the advance of this front. This may even be true in complex biomineralisation systems.

We considered both dilute polymer solutions and more concentrated ones. The most important findings of our simulations can be summarised as:

1. A single polymer has almost no effect on the velocity of the growth front of the condensed-matter phase.

2. Even a very weak attraction between the monomers of the polymer and the growth front is enough to drive incorporation of the polymer into the growing phase. This incorporation therefore occurs even when it is thermodynamically highly unfavourable. It is an intrinsically far-from-equilibrium process that relies on the slow dynamics of the polymer at the growth front interface.

3. Larger polymers create transient pits as the growth front grows around them. As the growth front passes the polymer, the growth rate is almost as fast as without the polymer. However, its sideways growth is very slow until the pit is quite deep, and this creates a steep-sided but transient pit in the growth front.

4. When the polymer concentration is high enough for the polymers to mostly cover the growth front, then polymers dramatically slow growth. 
5. When the polymer concentration is high enough to slow growth, and the polymers only bind to growth fronts along one axis, then the growth becomes highly anisotropic, and so the size of the domains that form changes from square to needle-like.

These results are all for homopolymers. Similar effects are seen for co-polymers (results not shown). If one of the blocks of a co-polymer attracts the growing phase, the polymer will tend stick to the growth front and be incorporated into the growing phase. Sufficiently high concentrations of co-polymers can also arrest growth.

Future experimental work could usefully study the behaviour of polymers as a function of their molecular weight. As the details of the monomer/surface interaction are not known for any polymer/crystal pair, interpreting data on polymers with different chemistries is difficult. However, varying the molecular weight keeps this interaction constant and so would allow a much 'cleaner' interpretation of the data, and so a more powerful experiment. Our results suggest that small oligomers would be excluded from the crystal but sufficiently large polymers would be included. This should be true providing the monomers of which they are made attract the crystal surface relatively weakly.

Diffusion of adsorbed single polymers at a surface can be studied in experiment, and the diffusion constant, $D_{S}$, measured ${ }^{29-31}$. If this could be done on a growing crystal surface, then the prediction is as follows. If the growth steps on the growth front move a distance equal to the polymer radius, $R_{G}$, in less time than the polymer takes to diffuse this distance, $R_{G S}^{2} / D_{S}$, then these steps will grow past and around a polymer. If the rate of advance of the growth front is also so fast that it creates and deepens a pit faster than the polymer can diffuse out of it, then the polymer will be incorporated into the growing crystal.

\section{Simulation method and model}

The model is based on a simple two-dimensional lattice gas on a square lattice. Without the polymer, the model is the lattice gas that is equivalent to the two-dimensional Ising model studied by Onsager ${ }^{32}$. Then each lattice site is either empty, or occupied by a single particle. Simulations are performed at constant chemical potential $\mu$. I write this as $\mu=\mu_{c o}+S$, where $\mu_{c o}$ is the chemical potential at coexistence, and $S$ is the supersaturation, i.e., the chemical potential driving force for growth. The lattice-gas particles attract each other via a nearest-neighbour interaction such that for every pair of particles on neighbouring lattice sites, the energy changes by $-\varepsilon$. Each site has four nearest neighbours. We work at fixed temperature $T$, and chemical potential $\mu$. Thus the number of particles (but not the number of polymers) varies. The correct Boltzmann weight of a state is $\exp (-U / k T+N \mu / k T)$. Here $U$ is the total energy and $N$ is the total number of lattice gas particles.

In the absence of polymer, the dynamics are Glauber dynamics, which are the standard implementation of the Metropolis Monte Carlo rule with non-conserved numbers of lattice-gas particles ${ }^{32,37}$. With these dynamics a lattice site is chosen at random, and the site is flipped from empty to occupied if it is empty, or from occupied to empty if it is occupied. If this increases the Boltzmann weight of the state the move is accepted, if it decreases the Boltzmann weight, the move is accepted with probability equal to the ratio of the new and old Boltzmann weights.

We use the Glauber algorithm because as it fixes the chemical potential it allows a 'clean' study of growth rates, without the complication of movement of the growth front changing the concentration profile near the growth front, and so changing the growth rate. Also, the growth rate is slow, see Fig. 2. This is because it occurs via nucleation and growth, as can be seen in Movie 1. Then as the rate is determined largely 
by the nucleation barrier, it depends only on weakly on diffusion. Thus, as the growth rate depends weakly on whether the microscopic dynamics are Glauber or diffusive, and as the results of Glauber dynamics are more straightforward to interpret, we use these dynamics.

A single homopolymer chain is modelled as a linear sequence of $L$ identical monomers, each of which occupies a single lattice site. Successive monomers along the polymer chain are constrained by bonds that limit the pair of successive monomers to be on either nearest-neighbour or next-nearest-neighbour sites, i.e., with monomer $m$ on site $\left(i_{m}, j_{m}\right)$, and monomer $m+1$ on site $\left(i_{m+1}, j_{m+1}\right)$, the bond enforces the constraint $\left(i_{m+1}-i_{m}\right)^{2}+\left(j_{m+1}-j_{m}\right)^{2} \leq 2$. Each site has 8 such neighbours. Neighbouring monomers do not interact except that a maximum of one monomer can occupy a site, i.e., the monomer-monomer interaction is just excluded volume. Therefore the polymer is in the "good solvent" regime, which is appropriate for modelling polymers that are highly water soluble. It also means that in the vapour phase of the lattice-gas model (our model for the aqueous solution), the polymer chain is swollen ${ }^{38}$ : the radius of gyration $R_{G} \simeq L^{3 / 4}$. A monomer attracts a neighbouring lattice-gas particle with an attraction of strength $\varepsilon_{M}$, i.e., for every neighbouring pair of a monomer and a particle the energy changes by $-\varepsilon_{M}$.

In the presence of a polymer, each move is either: 1) an attempt to move a single monomer; 2) an attempt to remove a lattice-gas particle from a filled site or add a lattice-gas particle to an empty site. Each move is with probability $f_{P}$, an attempt to move a monomer, and with probability $1-f_{P}$, an attempt to remove/add a particle. For a given simulation the number of monomers, $M_{P}$, is fixed, as is the number of sites that are not fixed, $M_{F L}$. Then the probability $f_{P}=s_{P} M_{P} /\left(s_{P} M_{P}+M_{F L}-M_{P}\right)$. Here $s_{P}$ is the ratio between the rate at which monomers move, and the rate at which sites fill or empty with lattice-gas particles. For almost all of our simulations we fix $s_{P}=10$, but we do vary $s_{P}$ in Fig. 2. Except for that figure $s_{P}=10$ always. This is fast enough to allow short weakly interacting polymers to be highly dynamic on the growth front, although strong interactions dramatically slow the dynamics of course. A cycle is defined as $\left(s_{P}-1\right) M_{P}+M_{F L}$ attempted moves, so that in one cycle we make one attempt on average to flip each of the $M_{F L}-M_{P}$ lattice sites not occupied by a monomer. We also make an average of $s_{P}$ attempts to move each of the $M_{P}$ monomers.

The dynamics of the polymer are as follows. When a monomer is selected, an attempt is made to move it to one of its 8 nearest or next-nearest neighbouring sites, selected at random. If the site selected to move the monomer is occupied, the move is rejected. If the site is vacant then the move is accepted if the move results in an increase in the Boltzmann weight. If it results in a decrease in the weight it is accepted with probability equal to the ratio of the new and old weights.

\subsection{Growth front simulations}

These are done in a simulation box with the bottom row of sites fixed in the occupied state and the top row fixed in the empty state, so here $M_{F L}=n_{L}\left(n_{L}-2\right)$. There are periodic boundary conditions along the horizontal direction. See Figs. 1, 3, 5 and 6 for snapshots from simulations of this type. One or more polymers are then introduced into the simulation. If it is one polymer then the polymer is placed with its lowest monomer a few sites above the bottom of the simulation box. When many polymers are required they are placed in a regular array in the simulation box. In all cases the polymers are simulated independently before placing in the simulation box to equilibrate their internal conformation. Then the simulation is run on and the condensed phase grows from the bottom row of sites fixed to be occupied, see for example Movie 1. The position of the growth front, required for the results of Fig. 2, is defined as being the top row of the lattice with more than $n_{L} / 2$ lattice-gas particles in it, i.e., the top layer that is more than half complete. 


\subsection{Anisotropic growth simulations}

These are done in a simulation box $n_{L}=500$ sites across. There are periodic boundary conditions along the horizontal and vertical axes. See Fig. 7 for a snapshot from a simulation of this type. We start with a square domain of the condensed phase 161 by 161 sites across surrounded by 700 polymers. This domain is significantly larger than the critical nucleus, ensuring that it will grow not shrink. Then the system is first partially equilibrated by running a simulation in which the lattice-gas particles are fixed but the polymers are moved for $10^{6}$ attempted moves per monomer. During this time polymers adsorb on the immobilised domain. Then once this is done the simulation is begun and the condensed-phase domain begins to grow.

For these simulations (and only these simulations) the interaction between a monomer and a lattice-gas particle (only) is made anisotropic. The interaction between a monomer and lattice-gas particle is $\varepsilon_{M} / \varepsilon=0.3$ along the horizontal axis, but is $\varepsilon_{M} / \varepsilon=-0.3$ along the vertical axis. Thus polymers bind to vertical growth fronts but do not bind to horizontal growth fronts, creating anisotropic growth. Of course if bonding is the same along both axes growth along both axes occurs at the same rate, with or without polymer.

\subsection{Thermodynamic equilibrium}

Here we will briefly consider the energetics and equilibrium behaviour of our system. For simplicity, let us consider the polymer in a completely extended configuration. If we bring a polymer in this configuration from the vapour phase to lie flat on the growth front, the energy change is $-L \varepsilon_{M}$. For $\varepsilon_{M}>0$, this is negative and it becomes very large for large $L$. However if we move the extended polymer from the vapour into the bulk of the condensed phase the energy change is $(2 L+2)\left(\varepsilon-\varepsilon_{M}\right)$. This is positive if $\varepsilon_{M}<\varepsilon$, and so is then unfavourable.

Here we study the regime where the polymer weakly binds to the condensed phase, i.e., where $0<$ $\varepsilon_{M}<\varepsilon$. Then at equilibrium, the polymer will be adsorbed at the interface and not as an inclusion in the condensed phase. The polymer is essentially a surfactant that has a minimum energy when at the interface. This implies that in this parameter range any incorporation in the growing condensed phase can only be due to non-equilibrium effects.

\section{Acknowledgements}

The simulations were inspired by experimental results of Kang Rae Cho working with Jim de Yoreo at the Laurence Berkeley National Laboratory, and Fiona Meldrum (University of Leeds).

\section{References}

1 S. Mann, Biomineralization, Oxford University Press, Oxford, 2001.

2 M. F. Ashby, L. J. Gibson, U. Wegst and R. Olive, Proc. Math. Phys. Sci., 1995, 450, 123.

3 M. Muthukumar, J. Chem. Phys., 2009, 130, 161101.

4 A. Herman, L. Addadi and S. Weiner, Nature, 1988, 331, 546.

5 F. C. Meldrum and H. Cölfen, Chem. Rev., 2008, 108, 4332. 
6 E. Bonucci, J. Bone Min. Metab., 2009, 27, 255-264.

7 C. Gilow, E. Zolotoyabko, O. Paris, P. Fratzl and B. Aichmayer, Crys. Growth Des., 2011, 11, 2054.

8 L. A. Estroff, L. Addadi, S. Weiner and A. D. Hamilton, Org. Biomol. Chem., 2004, 2, 137.

9 H. Li, H. L. Xin, D. A. Muller and L. A. Estroff, Science, 2009, 326, 1244.

10 H. Li and L. A. Estroff, Adv. Mat., 2009, 21, 470.

11 H. Li, Y. Fujiki, K. Sada and L. A. Estroff, CrystEngComm, 2011, 13, 1060.

12 Z. Deng, G. J. M. Habraken, M. Peeters, A. Heise, G. de With and N. A. J. M. Sommerdijk, Soft Matter, 2011, 7, 9685.

13 Y. Y. Kim, K. Ganesan, P. Yang, S. Kulak, A. N. Borukhin, S. Pechook, L. Ribeiro, K. R., S. J. Eichhorn, S. P. Armes, B. Pokroy and F. C. Meldrum, Nature Mat., 2011, 10, 890.

14 Y.-Y. Kim, L. Ribeiro, F. Maillot, O. Ward, S. J. Eichhorn and F. C. Meldrum, Adv. Mat., 2010, $22,2082$.

15 Y. Peng, A.-W. Xu, B. Deng, M. Antonietti and H. Cölfen, J. Phys. Chem. B, 2006, 110, 2988.

16 R. Kim, C. Kim, S. Lee, J. Kim and I. W. Kim, Crys. Growth Des., 2009, 9, 4584.

17 A. L. D. Vries and T. J. Price, Phil. Trans. Roy. Soc. London. Ser. B, Biol. Sci., 1984, 304, 575.

18 M. M. Tomczak, M. K. Gupta, L. F. Drummy, S. M. Rozenzhak and R. R. Naik, Acta Biomat., 2009, 5, 876.

19 R. W. Friddle, M. L. Weaver, S. R. Qiu, A. Wierzbicki, W. H. Casey and J. J. De Yoreo, Proc. Nat. Acad. Sci., 2010, 107, 11.

20 M.-K. Liang, O. Deschaume, S. V. Patwardhan and C. C. Perry, J. Mat. Chem., 2011, 21, 80.

21 L. Lakshtanov, N. Bovet and S. Stipp, Geochim. Cosmochim. Acta, 2011, 75, 3945 - 3955.

22 S. Weiner and L. Addadi, Ann. Rev. Mat. Res., 2011, 41, 21.

23 M. Seitz, C. Friedsam, W. Jöstl, T. Hugel and H. E. Gaub, ChemPhysChem, 2003, 4, 986.

24 A. P. Gunning, A. R. Kirby, A. R. MacKie, P. Kroon, G. Williamson and V. J. Morris, J. Micros., 2004, 216, 52.

25 D. Panja, G. T. Barkema and A. B. Kolomeisky, J. Phys.: Cond. Matt., 2009, 21, 242101.

26 H. E. Johnson and S. Granick, Science, 1992, 255, 966.

27 J. F. Douglas, H. M. Schneider, P. Frantz, R. Lipman and S. Granick, J. Phys.: Cond. Matt., 1997, 9, 7699.

28 K. Konstadinidis, S. Prager and M. Tirrell, J. Chem. Phys., 1992, 97, 7777. 
29 S. A. Sukhishvili, Y. Chen, J. D. Müller, E. Gratton, K. S. Schweizer and S. Granick, Nature, 2000, 406, year.

30 S. A. Sukhishvili, Y. Chen, J. D. Müller, E. Gratton, K. S. Schweizer and S. Granick, Macromolecules, 2002, 35, 1776.

31 J. S. S. Wong, L. Hong, S. C. Bae and S. Granick, Macromolecules, 2011, 44, 3073-3076.

32 D. Chandler, Introduction to Modern Statistical Mechanics, Oxford University Press, New York, 1987.

33 L. Onsager, Phys. Rev., 1944, 65, 117.

34 A. D. Sokal, in Monte Carlo and Molecular Dynamics Simulations in Polymer Science (edited by K. Binder), Oxford University Press, New York, 1995.

35 J. W. Mullin, Crystallization, Butterworth Heinemann, Oxford, 2001.

36 S.-H. Yu and H. Cölfen, J. Mat. Chem., 2004, 14, 2124.

37 K. Binder and D. W. Heermann, Monte Carlo Simulation in Statistical Physics: An Introduction, Springer Verlag, Heidelberg, 2010.

38 P.-G. de Gennes, Scaling Concepts in Polymer Physics, Cornell University Press, Ithaca, New York, 1979. 


\section{Supplementary Information}

Movie 1 This shows the dynamics of a growth front, without polymer. The movie shows one frame per 10 cycles, and 5 frames per second. In this and in all other movies, the condensed phase is red, the vapour white and the polymer is green. It is of a small, $n_{L}=30$, system so that growth is easier to see. The duration of the movie is 2,000 cycles. Note that for the much of the movie nothing happens. This is because the formation of a new layer is a nucleation-and-growth process and for much of the time, we are just waiting for a new layer to nucleate. Once a layer has nucleated it grows rapidly into a complete new monolayer. As it does so, the length of the layer fluctuates significantly, but, driven by the supersaturation, it eventually grows until the layer is complete. Then after a waiting time, a new layer nucleates and the process repeats. The parameters are $\varepsilon / k T=9$ and $S / k T=0.2$.

Movie 2 This shows the dynamics of a growth front, in the presence of polymer of length $L=10$. The movie shows one frame per 50 cycles, i.e., is speeded up in comparison to Movie 1 . It is of a system $n_{L}=100$ sites across but for clarity only a region of width 50 sites across is shown. The duration of the movie is 10,000 cycles. Note that when the polymer binds to the surface it blocks growth beneath but does not block growth outside the binding region, and so the growth front tends to grow past the polymer, leaving it in a deepening pit. Also note that as binding of the polymer to the growth front is not very strong $\left(\varepsilon_{M}=2.7 k T\right)$ occasionally monomers do unbind from the surface, and then the growth front advances into the space left by the unbinding monomers. The parameters are $\varepsilon / k T=9$, $\varepsilon_{M} / \varepsilon=0.3, S / k T=0.1$, and $s_{P}=10$.

Movie 3 This shows an $L=256$ polymer being incorporated into a growing phase. The duration of the movie is $2 \times 10^{5}$ cycles, The parameters are $\varepsilon / k T=9, \varepsilon_{M} / \varepsilon=0.3, S / k T=0.1, s_{P}=10$, and $n_{L}=400$.

Movie 4 This shows an $L=256$ polymer surfing along on the growth front. The duration of the movie is $10^{5}$ cycles, The parameters are $\varepsilon / k T=9, \varepsilon_{M} / \varepsilon=0.1, S / k T=0.1, n_{L}=400$ and $s_{P}=10$. The difference between this movie and Movie 3 is that the polymer only attracts the monomers of the growing phase very weakly.

Movie 5 This shows partially arrested growth in the presence of a high concentration of $L=10$ polymers. There are 225 polymers in a box $n_{L}=400$ sites across; only a part $200 \times 100$ sites across is shown. The interaction between a monomer and lattice-gas particle is $\varepsilon_{M} / \varepsilon=0.3$. The supersaturation $S / k T=0.1, \varepsilon / k T=9$ and $s_{P}=10$. The total duration of the movie is $10^{6}$ cycles.

Movie 6 This shows anisotropic growth in the presence of a high concentration of short $(L=10)$ polymers that adsorb onto the growth fronts along the horizontal axis but are repelled by the fronts along the vertical axis. There are 700 polymers in a box $n_{L}=500$ sites across. The interaction between a monomer and a lattice-gas particle is $\varepsilon_{M} / \varepsilon=0.3$ along the horizontal axis but is $\varepsilon_{M} / \varepsilon=-0.3$ along the vertical axis. The supersaturation $S / k T=0.1, \varepsilon / k T=9$, and $s_{P}=10$. The total duration of the movie is 100,000 cycles, and it is 100 frames at 5 frames per second. 